\title{
Auditory Hallucination
}

National Cancer Institute

\section{Source}

National Cancer Institute. Auditory Hallucination. NCI Thesaurus. Code C118174.

Perception of sound in the absence of a corresponding stimulus. 